\title{
ON THE LINK BETWEEN DEPENDENCE AND INDEPENDENCE IN EXTREME VALUE THEORY FOR DYNAMICAL SYSTEMS
}

\author{
ANA CRISTINA MOREIRA FREITAS AND JORGE MILHAZES FREITAS
}

\begin{abstract}
It is well known that under some conditions on the dependence structure we can relate the asymptotic distribution of the partial maximum of a stationary stochastic process with the maximum of an associated independent sequence of random variables with the same distribution function of the dependent one. These conditions are known as $D\left(u_{n}\right)$ and $D^{\prime}\left(u_{n}\right)$. Although $D\left(u_{n}\right)$ is of mixing type, when studying stochastic processes arising from a dynamical system with good mixing properties, verifying $D\left(u_{n}\right)$ is not straightforward. We propose a reformulation of $D\left(u_{n}\right)$ so that its validity may follow easily if we have a certain decay of correlations for the dynamical system in consideration.
\end{abstract}

\section{INTRODUCTION}

Let $(\Omega, \mathcal{A}, P)$ be a probability space and $X: \Omega \rightarrow \mathbb{R}$ a random variable (r.v.) with distribution function (d.f.) given by $F(x)=P(X \leq x)$. Also, let $E(\cdot)$ denote expectation with respect to $P$ so that $E(X)=\int X d P=\int x d F(x)$. Consider a stationary stochastic process $X_{0}, X_{1} \ldots$ of r.v. defined on $\Omega$ with common d.f. $F$. For notational simplicity we assume that $X=X_{0}$. For every $i, j, n \in \mathbb{N}$, set

$$
M_{i, j}:=\max \left\{X_{i}, X_{i+1}, \ldots, X_{i+j-1}\right\} \text { and } M_{n}:=M_{0, n} .
$$

The study of the limiting behavior for maxima of a stationary process can be reduced, under adequate conditions on the dependence structure, to the Classical Extreme Value Theory for sequences of i.i.d. r.v. Hence, to the stationary process $X_{0}, X_{1}, \ldots$ we associate an independent sequence of r.v. denoted by $Z_{0}, Z_{1}, \ldots$ with common d.f. $F$. We also set for each $n \in \mathbb{N}$

$$
\hat{M}_{n}:=\max \left\{Z_{0}, \ldots, Z_{n-1}\right\} .
$$

Let us focus on the conditions that allow us to relate the asymptotic distribution of $M_{n}$ with that of $\hat{M}_{n}$. Following Leadbetter et al. (1983) we refer to these conditions as $D\left(u_{n}\right)$ and $D^{\prime}\left(u_{n}\right)$, where $u_{n}$ is a suitable sequence of thresholds converging to $\sup _{\omega \in \Omega} X(\omega)=1$, as $n$ goes to $\infty$, that will be defined below. $D\left(u_{n}\right)$ imposes a certain type of distributional mixing property. Essentially, it says that the dependence between some special type of

Date: January 11, 2008.

2000 Mathematics Subject Classification. 37A50, 37C40, 60G10, 60G70.

Key words and phrases. Dependence, Extreme Value Theory, Stationary Stochastic Processes, Ergodic Theory, Decay of Correlations. 
events fades away as they become more and more apart in the time line. $D^{\prime}\left(u_{n}\right)$ restricts the occurrence of a large number of 'exceedances' of the level $u_{n}$ close together in time.

The purpose of this work is to verify that condition $D\left(u_{n}\right)$ can be stated in a weaker form and the result still prevails. The advantage of having this weaker requirement is that, in the context of Dynamical Systems, $D\left(u_{n}\right)$ should follow from decay of correlations.

We say that an exceedance of level $u_{n}$ occurs at time $i$ if $X_{i}>u_{n}$. The probability of such an exceedance is $1-F\left(u_{n}\right)$ and so the mean value of the number of exceedances occurring up to $n$ is $n\left(1-F\left(u_{n}\right)\right)$. The sequences of levels $u_{n}$ we consider are such that $n\left(1-F\left(u_{n}\right)\right) \rightarrow \tau$ as $n \rightarrow \infty$, for some $\tau \geq 0$, which means that, in a time period of length $n$, the expected number of exceedances is approximately $\tau$.

Condition $D\left(u_{n}\right)$ is a type of mixing requirement specially adapted to Extreme Value Theory. In this context, the events of interest are those of the form $\left\{X_{i} \leq u\right\}$ and their intersections. Observe that $\left\{M_{n} \leq u\right\}$ is just $\left\{X_{0} \leq u, \ldots, X_{n-1} \leq u\right\}$. Motivated by the work of Collet (2001), we propose:

Condition $\left(D\left(u_{n}\right)\right)$. We say that $D\left(u_{n}\right)$ holds for the sequence $X_{0}, X_{1}, X_{2}, \ldots$ if for any integers $\ell, t$ and $n$

$$
P\left(\left\{X_{0}>u_{n}\right\} \cap\left\{M_{t, \ell} \leq u_{n}\right\}\right)-P\left(\left\{X_{0}>u_{n}\right\}\right) P\left(\left\{M_{\ell} \leq u_{n}\right\}\right) \leq \gamma(n, t),
$$

where $\gamma(n, t)$ is nonincreasing in $t$ for each $n$ and $n \gamma\left(n, t_{n}\right) \rightarrow 0$ as $n \rightarrow \infty$ for some sequence $t_{n}=o(n)$, which means that $t_{n} / n \rightarrow 0$ as $n \rightarrow \infty$.

We remark that the actual definition of $D\left(u_{n}\right)$ appearing in Leadbetter et al. (1983), Section 3.2, is, in some sense, a stronger requirement.

The sequence $u_{n}$ is such that the average number of exceedances in the time interval $\{0, \ldots,[n / k]\}$ is approximately $\tau / k$, which goes to zero as $k \rightarrow \infty$. However, the exceedances may have a tendency to be concentrated in the time period following the first exceedance at time 0 . To avoid this we introduce

Condition $\left(D^{\prime}\left(u_{n}\right)\right)$. We say that $D^{\prime}\left(u_{n}\right)$ holds for the sequence $X_{0}, X_{1}, X_{2}, \ldots$ if

$$
\lim _{k \rightarrow \infty} \limsup _{n \rightarrow \infty} n \sum_{j=1}^{[n / k]} P\left(\left\{X_{0}>u_{n}\right\} \cap\left\{X_{j}>u_{n}\right\}\right)=0 .
$$

Notice that Condition 1.3 forbids the concentration of exceedances by bounding the probability of more than one exceedance in the time interval $\{0, \ldots,[n / k]\}$. This guarantees that the exceedances should appear scattered through the time period $\{0, \ldots, n-1\}$.

Our goal is to show that $M_{n}$ and $\hat{M}_{n}$ have the same asymptotic distribution under $D\left(u_{n}\right)$ and $D^{\prime}\left(u_{n}\right)$.

Theorem 1. Let $\left(u_{n}\right)_{n \in \mathbb{N}}$ be such that $n P\left(X>u_{n}\right)=n\left(1-F\left(u_{n}\right)\right) \rightarrow \tau$, as $n \rightarrow \infty$, for some $\tau \geq 0$. Assume that conditions $D\left(u_{n}\right)$ and $D^{\prime}\left(u_{n}\right)$ hold. Then

$$
\lim _{n \rightarrow \infty} P\left(M_{n} \leq u_{n}\right)=\lim _{n \rightarrow \infty} P\left(\hat{M}_{n} \leq u_{n}\right) .
$$




\section{Extremes on Dynamical Systems}

In this section, we observe that the study of the partial maximum of deterministic processes arising from a certain dynamical system that possesses an invariant measure, with sufficient decay of correlations, can be reduced to proving $D^{\prime}\left(u_{n}\right)$ and investigating the domain of attraction of the maximum of the associated independent process.

Let $f: M \rightarrow M$ be a smooth map on the manifold $M$ admitting an invariant Borel probability measure $\mu$. Assume that there exists a nonincreasing $g: \mathbb{N} \rightarrow \mathbb{R}$ such that for all $\varphi, \psi: M \rightarrow \mathbb{R}$ with bounded variation, there is $C>0$ independent of $\varphi, \psi$ and $n$ such that

$$
\left|\int \varphi \cdot\left(\psi \circ f^{n}\right) d \mu-\int \varphi d \mu \int \psi d \mu\right| \leq C \operatorname{Var}(\varphi)\|\psi\|_{\infty} g(n), \quad \forall n \geq 0,
$$

where $\operatorname{Var}(\varphi)$ denotes the total variation of $\varphi$ and $n g\left(t_{n}\right) \rightarrow 0$, as $n \rightarrow \infty$ for some $\left(t_{n}\right)_{n \in \mathbb{N}}$ such that $t_{n}=o(n)$.

Consider now the r.v. $X: M \rightarrow \mathbb{R}$ of bounded variation on the probability space $(M, \mathcal{B}, \mu)$, where $\mathcal{B}$ is the Borel $\sigma$-algebra on $M$, with d.f. $F(x)=\mu\left(\left\{X^{-1}(-\infty, x]\right\}\right)$. Define the stationary stochastic process $X_{0}, X_{1}, X_{2}, \ldots$ by $X_{i}=X \circ f^{i}$. Let

$$
M_{n}=\max \left\{X_{0}, \ldots, X_{n-1}\right\},
$$

for each $n \in \mathbb{N}$. Denote by $Z_{0}, Z_{1}, Z_{2}, \ldots$ an independent sequence of r.v. with common d.f. $F$ and set, for each $n \in \mathbb{N}$,

$$
\hat{M}_{n}=\max \left\{Z_{0}, \ldots, Z_{n-1}\right\} .
$$

Taking $\varphi=\mathbf{1}_{\left\{X>u_{n}\right\}}$ and $\psi=\mathbf{1}_{\left\{M_{\ell} \leq u_{n}\right\}}$, then (2.1) implies that Condition $D\left(u_{n}\right)$ holds with $\gamma(n, t)=\gamma(t)=C \operatorname{Var}\left(\mathbf{1}_{\left\{X>u_{n}\right\}}\right)\left\|\mathbf{1}_{\left\{M_{\ell} \leq u_{n}\right\}}\right\|_{\infty} g(t) \leq C^{\prime} \operatorname{Var}(X) g(t)$ and for the sequence $t_{n}$ such that $t_{n} / n \rightarrow 0$ and $n g\left(t_{n}\right) \rightarrow 0$ as $n \rightarrow \infty$.

This means that if we are also able to prove $D^{\prime}\left(u_{n}\right)$ for the sequence $X_{0}, X_{1}, \ldots$ then the study of the limiting behavior of $M_{n}$ is reduced to study the domain of attraction for the maxima of $F$ using the usual tools of Classical Extreme Value Theory, available, for example, in Section 1.6 of Leadbetter et al. (1983). This type of study, namely the proof of condition $D^{\prime}\left(u_{n}\right)$, was proved in the context of Benedicks-Carleson quadratic dynamical systems, in Freitas and Freitas (2007). We also mention the work of Haiman (2003) who obtained an extreme limiting law for the tent map using an approach relying on the linear property of the map.

Remark 2. Observe that often decay of correlations is know for Hölder continuous functions instead. In these situations one can use an Hölder continuous approximation of $\mathbf{1}_{\left\{X>u_{n}\right\}}$ and try to prove that the error introduced does not interfere too much, as Collet (2001) did in Lemma 3.3.

\section{Proof of the Result}

In this section, we prove Theorem 1 using the same type of approach used in Section 3 of Collet (2001). We begin by stating two abstract Lemmas. 
Lemma 3.1. For any $\ell \in \mathbb{N}$ and $u \in \mathbb{R}$ we have

$$
\sum_{j=0}^{\ell-1} P\left(X_{j}>u\right) \geq P\left(M_{\ell}>u\right) \geq \sum_{j=0}^{\ell-1} P\left(X_{j}>u\right)-\sum_{j=0}^{\ell-1} \sum_{i \neq j, i=0}^{\ell-1} P\left(\left\{X_{j}>u\right\} \cap\left\{X_{i}>u\right\}\right)
$$

Proof. This is a straightforward consequence of the formula for the probability of a multiple union on events. See for example first Theorem of Chapter 4 in Feller (1952).

Lemma 3.2. Assume that $t, r, m, \ell, s$ are nonnegative integers. Then, we have

$$
0 \leq P\left(M_{r} \leq u\right)-P\left(M_{r+\ell} \leq u\right) \leq \ell \cdot P(X>u)
$$

and

$$
\begin{aligned}
\mid P\left(M_{s+t+m} \leq u\right)-P\left(M_{m} \leq u\right) & +\sum_{j=0}^{s-1} P\left(\{X>u\} \cap\left\{M_{s+t-j, m} \leq u\right\}\right) \mid \\
& \leq 2 s \sum_{j=1}^{s-1} P\left(\{X>u\} \cap\left\{X_{j}>u\right\}\right)+t P(X>u) .
\end{aligned}
$$

Proof. Obviously,

$$
0 \leq P\left(M_{r} \leq u\right)-P\left(M_{r+\ell} \leq u\right)=\sum_{j=0}^{\ell-1}\left(P\left(M_{r+j} \leq u\right)-P\left(M_{r+j+1} \leq u\right)\right) .
$$

By the law of total probability we have, for any $i \geq 0$,

$$
P\left(M_{i} \leq u\right)=P\left(M_{i+1} \leq u\right)+P\left(\left\{M_{i} \leq u\right\} \cap\left\{X_{i}>u\right\}\right) \leq P\left(M_{i+1} \leq u\right)+P\left(X_{i}>u\right)
$$

and the first statement of the Lemma follows by stationarity.

For the second statement observe that

$$
\left\{M_{s+t+m} \leq u\right\}=\left\{M_{s} \leq u\right\} \cap\left\{M_{s, t} \leq u\right\} \cap\left\{M_{s+t, m} \leq u\right\} .
$$

Consequently,

$$
\left(\left\{M_{s} \leq u\right\} \cap\left\{M_{s+t, m} \leq u\right\}\right) \backslash\left\{M_{s+t+m} \leq u\right\} \subset\left\{M_{s, t}>u\right\} .
$$

Thus, using the first inequality of Lemma 3.1 we obtain

$$
\left|P\left(\left\{M_{s} \leq u\right\} \cap\left\{M_{s+t, m} \leq u\right\}\right)-P\left(M_{s+t+m} \leq u\right)\right| \leq t P(X>u) .
$$

Using stationarity and the first inequality in Lemma 3.1 we have

$$
\begin{aligned}
P\left(\left\{M_{s} \leq u\right\} \cap\left\{M_{s+t, m} \leq u\right\}\right) & =P\left(M_{s+t, m} \leq u\right)-P\left(\left\{M_{s}>u\right\} \cap\left\{M_{s+t, m} \leq u\right\}\right) \\
& =P\left(M_{m} \leq u\right)-P\left(\left\{M_{s}>u\right\} \cap\left\{M_{s+t, m} \leq u\right\}\right) \\
& \geq P\left(M_{m} \leq u\right)-\sum_{j=0}^{s-1} P\left(\left\{X_{j}>u\right\} \cap\left\{M_{s+t, m} \leq u\right\}\right) .
\end{aligned}
$$


LINK BETWEEN DEPENDENCE AND INDEPENDENCE IN THE EXTREME VALUE THEORY 5

Now, by the second inequality in Lemma 3.1 and stationarity we have

$$
\begin{aligned}
P\left(\left\{M_{s} \leq u\right\} \cap\left\{M_{s+t, m} \leq u\right\}\right) \leq & P\left(M_{m} \leq u\right)-\sum_{j=0}^{s-1} P\left(\left\{X_{j}>u\right\} \cap\left\{M_{s+t, m} \leq u\right\}\right) \\
& +\sum_{j=0}^{s-1} \sum_{j \neq \ell, \ell=0}^{s-1} P\left(\left\{X_{j}>u\right\} \cap\left\{X_{\ell}>u\right\} \cap\left\{M_{s+t, m} \leq u\right\}\right) .
\end{aligned}
$$

Finally, stationarity and the last three inequalities give

$$
\begin{array}{r}
\left|P\left(\left\{M_{s} \leq u\right\} \cap\left\{M_{s+t, m} \leq u\right\}\right)-P\left(M_{m} \leq u\right)+\sum_{j=0}^{s-1} P\left(\{X>u\} \cap\left\{M_{s+t-j, m} \leq u\right\}\right)\right| \\
\leq 2 s \sum_{j=1}^{s-1} P\left(\{X>u\} \cap\left\{X_{j}>u\right\}\right),
\end{array}
$$

and the result follows by (3.3).

Proof of Theorem 1. Let $\ell=\ell_{n}=\left[\frac{n}{k}\right]$ where $\left[\frac{n}{k}\right]$ is the integer part of $\frac{n}{k}$. We begin by replacing $P\left(M_{n} \leq u_{n}\right)$ by $P\left(M_{k(\ell+t)} \leq u_{n}\right)$ for some $t>1$. According to (3.1) of Lemma 3.2, we have

$$
\left|P\left(M_{n} \leq u_{n}\right)-P\left(M_{k(\ell+t)} \leq u_{n}\right)\right| \leq k t P\left(X>u_{n}\right) .
$$

We now estimate recursively $P\left(M_{i(\ell+t)} \leq u_{n}\right)$ for $i=0, \ldots, k$. Using (3.2) of Lemma 3.2 and stationarity, we have for any $1 \leq i \leq k$

$$
\left|P\left(M_{i(\ell+t)} \leq u_{n}\right)-\left(1-\ell P\left(X>u_{n}\right)\right) P\left(M_{(i-1)(\ell+t)} \leq u_{n}\right)\right| \leq \Gamma_{n, i},
$$

where

$$
\begin{aligned}
\Gamma_{n, i}= & \left|\ell P\left(X>u_{n}\right) P\left(M_{(i-1)(\ell+t)} \leq u_{n}\right)-\sum_{j=0}^{\ell-1} P\left(\left\{X_{j}>u_{n}\right\} \cap\left\{M_{\ell+t,(i-1)(\ell+t)} \leq u_{n}\right\}\right)\right| \\
& +t P\left(X>u_{n}\right)+2 \ell \sum_{j=1}^{\ell-1} P\left(\left\{X>u_{n}\right\} \cap\left\{X_{j}>u_{n}\right\}\right) .
\end{aligned}
$$

Using stationarity, $D\left(u_{n}\right)$ and, in particular, that $\gamma(n, t)$ is nonincreasing in $t$ for each $n$ we conclude

$$
\begin{aligned}
\Gamma_{n, i} \leq & \sum_{j=0}^{\ell-1}\left|P\left(X_{0}>u_{n}\right) P\left(M_{(i-1)(\ell+t)} \leq u_{n}\right)-P\left(\left\{X_{0}>u_{n}\right\} \cap\left\{M_{\ell+t-j,(i-1)(\ell+t)} \leq u_{n}\right\}\right)\right| \\
& +t P\left(X>u_{n}\right)+2 \ell \sum_{j=1}^{\ell-1} P\left(\left\{X>u_{n}\right\} \cap\left\{X_{j}>u_{n}\right\}\right) \\
\leq & \ell \gamma(n, t)+t P\left(X>u_{n}\right)+2 \ell \sum_{j=1}^{\ell-1} P\left(\left\{X>u_{n}\right\} \cap\left\{X_{j}>u_{n}\right\}\right) .
\end{aligned}
$$


Define $\Upsilon_{n}=\ell \gamma(n, t)+t P\left(X>u_{n}\right)+2 \ell \sum_{j=1}^{\ell-1} P\left(\left\{X>u_{n}\right\} \cap\left\{X_{j}>u_{n}\right\}\right)$. Then for every $1<i \leq k$ we have

$$
\left|P\left(M_{i(\ell+t)} \leq u_{n}\right)-\left(1-\ell P\left(X>u_{n}\right)\right) P\left(M_{(i-1)(\ell+t)} \leq u_{n}\right)\right| \leq \Upsilon_{n}
$$

and for $i=1$

$$
\left|P\left(M_{(\ell+t)} \leq u_{n}\right)-\left(1-\ell P\left(X>u_{n}\right)\right)\right| \leq \Upsilon_{n}
$$

Assume that $k$ and $n$ are large enough in order to have $\ell P\left(X>u_{n}\right)<2$, which implies that $\left|1-\ell P\left(X>u_{n}\right)\right|<1$. A simple inductive argument allows to conclude

$$
\left|P\left(M_{k(\ell+t)} \leq u_{n}\right)-\left(1-\ell P\left(X>u_{n}\right)\right)^{k}\right| \leq k \Upsilon_{n} .
$$

Recalling (3.4), we have

$$
\left|P\left(M_{n} \leq u_{n}\right)-\left(1-\ell P\left(X>u_{n}\right)\right)^{k}\right| \leq k t P\left(X>u_{n}\right)+k \Upsilon_{n} .
$$

Since $n P\left(X>u_{n}\right)=n\left(1-F\left(u_{n}\right)\right) \rightarrow \tau$, as $n \rightarrow \infty$, for some $\tau \geq 0$, we have

$$
\lim _{k \rightarrow \infty} \lim _{n \rightarrow \infty}\left(1-\left[\frac{n}{k}\right] P\left(X>u_{n}\right)\right)^{k}=\lim _{k \rightarrow \infty}\left(1-\frac{\tau}{k}\right)^{k}=\mathrm{e}^{-\tau} .
$$

Now, observe that $n P\left(X>u_{n}\right)=n\left(1-F\left(u_{n}\right)\right) \rightarrow \tau$ is equivalent to $P\left(\hat{M}_{n} \leq u_{n}\right)=$ $\left(F\left(u_{n}\right)\right)^{n} \rightarrow \mathrm{e}^{-\tau}$, where the limits are taken when $n \rightarrow \infty$ and $\tau \geq 0$ (see Leadbetter et al. (1983), Theorem 1.5.1] for a proof of this fact). Hence,

$$
\lim _{k \rightarrow \infty} \lim _{n \rightarrow \infty}\left(1-\left[\frac{n}{k}\right] P\left(X>u_{n}\right)\right)^{k}=\lim _{n \rightarrow \infty} P\left(\hat{M}_{n} \leq u_{n}\right) .
$$

It is now clear that, according to (3.5) and (3.6), $M_{n}$ and $\hat{M}_{n}$ share the same limiting distribution if

that is

$$
\lim _{k \rightarrow \infty} \lim _{n \rightarrow \infty}\left(k t P\left(X>u_{n}\right)+k \Upsilon_{n}=0\right.
$$

$$
\lim _{k \rightarrow \infty} \lim _{n \rightarrow \infty} 2 k t P\left(X>u_{n}\right)+n \gamma(n, t)+2 n \sum_{j=1}^{\ell} P\left(\left\{X>u_{n}\right\} \cap\left\{X_{j}>u_{n}\right\}\right)=0 .
$$

Assume that $t=t_{n}$ where $t_{n}=o(n)$ is given by Condition $D\left(u_{n}\right)$. Then, for every $k \in \mathbb{N}$, we have $\lim _{n \rightarrow \infty} k t_{n} P\left(X>u_{n}\right)=0$, since $n P\left(X>u_{n}\right) \rightarrow \tau \geq 0$. Finally, we use $D\left(u_{n}\right)$ and $D^{\prime}\left(u_{n}\right)$ to obtain that the two remaining terms in (3.7) also go to 0 .

\section{REFERENCES}

Collet, P., 2001. Statistics of closest return for some non-uniformly hyperbolic systems. Ergd. Th. \& Dynam. Sys. 21 (2), 401-420.

Feller, W., 1952. An introduction to Probability Theory and its Applications, Volume I. Wiley Publications in Statistics.

Freitas, J.M. and Freitas, A.C.M. Extreme values for Benedicks-Carleson quadratic maps. To appear in Ergd. Th. \& Dynam. Sys.

Haiman, G., 2003. Extreme values of the tent map process. Stat. Probab. Lett., 65 (4), 451-456.

Leadbetter, M.R., Lindgren, G. and Rootzén, H., 1983. Extremes and related properties of stationary sequences and processes. Springer Series in Statistics, Springer: New York. 
Ana Cristina Moreira Freitas, Centro de Matemática \& Faculdade de Economia da Universidade do Porto, Rua Dr. Roberto Frias, 4200-464 Porto, Portugal

E-mail address: amoreira@fep.up.pt

Jorge Milhazes freitas, Centro de Matemática da Universidade do Porto, Rua do Campo Alegre 687, 4169-007 Porto, Portugal

E-mail address: jmfreita@fc.up.pt

$U R L:$ http://www.fc.up.pt/pessoas/jmfreita 